\title{
Some cautions on the use of the LLC panel unit root test
}

Citation for published version (APA):

Westerlund, J. (2006). Some cautions on the use of the LLC panel unit root test. METEOR, Maastricht University School of Business and Economics. METEOR Research Memorandum No. 055

https://doi.org/10.26481/umamet.2006055

Document status and date:

Published: 01/01/2006

DOI:

10.26481/umamet.2006055

Document Version:

Publisher's PDF, also known as Version of record

\section{Please check the document version of this publication:}

- A submitted manuscript is the version of the article upon submission and before peer-review. There can be important differences between the submitted version and the official published version of record.

People interested in the research are advised to contact the author for the final version of the publication, or visit the DOI to the publisher's website.

- The final author version and the galley proof are versions of the publication after peer review.

- The final published version features the final layout of the paper including the volume, issue and page numbers.

Link to publication

\footnotetext{
General rights rights.

- You may freely distribute the URL identifying the publication in the public portal. please follow below link for the End User Agreement:

www.umlib.nl/taverne-license

Take down policy

If you believe that this document breaches copyright please contact us at:

repository@maastrichtuniversity.nl

providing details and we will investigate your claim.
}

Copyright and moral rights for the publications made accessible in the public portal are retained by the authors and/or other copyright owners and it is a condition of accessing publications that users recognise and abide by the legal requirements associated with these

- Users may download and print one copy of any publication from the public portal for the purpose of private study or research.

- You may not further distribute the material or use it for any profit-making activity or commercial gain

If the publication is distributed under the terms of Article $25 \mathrm{fa}$ of the Dutch Copyright Act, indicated by the "Taverne" license above, 
Joakim Westerlund

Some Cautions on the Use of the LLC Panel Unit Root Test

$\mathrm{RM} / 06 / 055$

JEL code : C12, C23

\section{METE@R}

Maastricht research school of Economics of TEchnology and ORganizations

Universiteit Maastricht

Faculty of Economics and Business Administration P.O. Box 616

NL - 6200 MD Maastricht

phone : :+31433883830

fax $\quad$ : ++31433884873 



\title{
Some Cautions on the Use of the LLC Panel Unit Root Test*
}

\author{
Joakim Westerlund ${ }^{\dagger}$
}

November 5, 2006

\begin{abstract}
One of the single most cited studies within the field of nonstationary panel data analysis is that of LLC (Levin, Lin and Chu, 2002. Unit Root Tests in Panel Data: Asymptotic and Finite Sample Properties. Journal of Econometrics 98, 1-24), in which the authors propose a test for a common unit root in the panel. Using both theoretical arguments and simulation evidence, we show that this test generally suffers from serious bias when combined with most commonly used rules for lag length and bandwidth selection. To remedy this bias effect, we propose a slightly modified test that performs well in small samples and that is computationally more convenient than the LLC test.
\end{abstract}

JEL Classification: C12; C23.

Keywords: Panel Data; Unit Root Test.

\section{Introduction}

During the last few years, it has become routine to test for the presence of unit roots when working with panel data. One of the most widely applied tests within this field of research is that of LLC, which is designed to test the null hypothesis of a common unit root in the panel versus the alternative of stationarity when the cross-sectional units are independent of each other. Some

\footnotetext{
* The author would like to thank Denis de Crombrugghe, Bernd Fitzenberger, Rolf Larsson, Franz Palm, Jean-Pierre Urbain, three anonymous referees, and seminar participants at Lund and Maastricht university for many valuable comments and suggestions. The author would also like to thank the Maastricht Research School of Economics of Technology and Organizations for its hospitality during a visit at the Depertment of Quantitative Economics at the University of Maastricht, where a part of this paper was written. Thank you also to the Jan Wallander and Tom Hedelius Foundation for financial support under research grant number W2006-0068:1. The usual disclaimer applies.

${ }^{\dagger}$ Department of Economics, Lund University, P. O. Box 7082, S-220 07 Lund, Sweden. Telephone: +46 46222 4970; Fax: +46 46222 4118; E-mail address: joakim.westerlund@nek.lu.se.
} 
examples of recently published studies that have applied this test include Kim et al. (2005) for international capital mobility, Gutierrez and Gutierrez (2003) for international R\&D spillover effects, Crespo-Cuaresma et al. (2005) for monetary exchange rate modelling, and Jenkins and Snaith (2005) and Basher and Mohsin (2004) for purchasing power parity, to mention a few.

The LLC study has not only been important among applied researchers but also among econometricians concerned with the development of new tests and methods for nonstationary panel data. One particularly fruitful area, in which the LLC test has been instrumental, is that of cross-sectionally dependent panels. Examples of studies that have attempted extend the LLC test in this direction include O'Connell (1998), Moon and Perron (2004), and most recently Jönsson (2005) and Breitung and Das (2005). Another important extension of the LLC test is provided by Kao (1999), who considers the problem of testing for cointegration in panel data.

It is clear that the LLC study has played a key role in most, if not all, areas of research concerned with nonstationary panel data. Because of this, the study has also been subject to much scrutiny. For example, the studies of Maddala and Wu (1999), Karlsson and Löthgren (1999), O'Connell (1998), Jönsson (2005) and Hlouskova and Wagner (2006) have all contributed by evaluating the smallsample properties of the LLC test. The overall impression being that the test performs well with good size and power under the assumptions spelled out by LLC.

In this paper, we point to a weakness in the LLC test that seems to have been largely overlooked in the earlier literature. ${ }^{1}$ In particular, by using both theoretical arguments and simulation evidence, we demonstrate that the correction employed by LLC to account for the nonzero mean of their test statistic in the presence of deterministic intercept and trend terms can have very adverse side effects when combined with commonly used lag length and kernel bandwidth selection rules, such as those proposed by Campbell and Perron (1991), and Newey and West (1994), respectively. In fact, our simulation evidence reveals that the power of the test can be extremely poor, and practically nonexisting, unless the lag and bandwidth parameters are chosen exactly as in LLC.

To alleviate these unwanted side effects, we propose a slightly modified test that does not have the same drawbacks as the original LLC test, and that is computationally much more convenient. Our simulation results show that the modified test has small size distortions and vastly superior power in comparison to the original test. To illustrate the empirical significance of these findings, we consider as an example the rate of inflation.

The paper proceeds as follows. In the next three sections, we describe the problem under consideration and develop a modified test that circumvents it. Section 5 is then devoted to the simulation study while Section 6 contains the empirical illustration. Section 7 concludes.

\footnotetext{
${ }^{1}$ In fact, the only study that we are aware of that mentions this is that of Breitung (2000).
} 


\section{Model and assumptions}

In this section, we briefly outline the data generating process employed by LLC, which will also form the basis for our modified test. In particular, it is assumed that the multidimensional time series variable $y_{i t}$ is generated as

$$
\Delta y_{i t}=\alpha_{i} d_{t}+\delta y_{i t-1}+u_{i t}
$$

where $i=1, \ldots, T$ and $i=1, \ldots, N$ indexes the time series and cross-sectional units, respectively. For the deterministic component, here denoted by $d_{t}$, we have three specifications. In Model $1, d_{t}=0$, in Model $2, d_{t}=1$ and in Model $3, d_{t}=(1, t)^{\prime}$. It is further assumed that the individual error processes $u_{i t}$ are independent, and that they follow a stationary and invertible autoregressive (AR) process.

Assumption 1. (Error process.) The process $u_{i t}$ satisfies the following set of conditions:

(a) $E\left(u_{i t} u_{k j}\right)=0$ for all $i \neq k, t$ and $j$;

(b) $\phi_{i}(L) u_{i t}=e_{i t}$, where $\phi_{i}(L)=1-\sum_{j=1}^{P_{i}} \phi_{i j} L^{j}$ is a polynomial in the lag operator $L$ and $e_{i t}$ is independent and identically distributed with mean zero and variance $\sigma_{i}^{2}<\infty$.

To be able to derive the asymptotic theory, we also require the following condition.

Assumption 2. (Invariance principle.) $T^{-1 / 2} \sum_{t=1}^{[r T]} e_{i t} \Rightarrow \sigma_{i} W_{i}(r)$ as $T \rightarrow \infty$ for each $i$, where $\Rightarrow$ denotes weak convergence and $W_{i}(r)$ is a standard Brownian motion defined on the unit interval $r \in[0,1]$.

Assumptions 1 and 2 establish the basic conditions underlying the LLC test. These will prove very important in the sequel, and it is therefore instructive to consider briefly their implications. Take Assumption 1 (a). This type of independence assumption is typical for our panel approach and we will use it here in the derivation of the asymptotic distribution of our modified test statistic. In applied work, however, it may be useful to be able to allow for at least some kind of dependence among the cross-sectional units. A very straightforward and common way to do this is to assume that the dependence can be approximated by means of common time effects, which do not affect the limiting distribution of the test.

Assumption 1 (b) is also very standard and states that each of the individual processes $u_{i t}$ follows an $\mathrm{AR}$ process of possibly infinite order $P_{i}$, which means that the data can be serially correlated in a very general way. Note also that, although we have assumed a common value $\delta$ for the AR parameter, all other parameters in the error process are permitted to vary freely across the members of the panel. In particular, note how the serial correlation of $u_{i t}$ is permitted to 
vary across $i$ through its long-run variance

$$
\omega_{i}^{2}=\frac{\sigma_{i}^{2}}{\phi_{i}(1)^{2}} .
$$

As will be shown later, this allowance make the construction of the LLC test quite complicated.

Assumption 2 states that an invariance principle applies to the partial sum process constructed from $e_{i t}$ as $T$ grows for a given $i$. This assumption is convenient for at least two reasons. First, it makes the asymptotic analysis relatively uncomplicated. Second, apart from some mild regulatory conditions, it places very little restrictions on the time series properties of the data generating process.

\section{The LLC test}

The hypothesis to be tested is that all the members of the panel have a unit root against the alternative that all the members are stationarity, which is equivalent to testing $H_{0}: \delta=0$ against $H_{1}: \delta<0$. Under the null and Assumption 1 (b), by simply substituting for $u_{i t}$ in (1), this can be done by using the following augmented test regression

$$
\Delta y_{i t}=\delta y_{i t-1}+\sum_{j=1}^{P_{i}} \phi_{i j} \Delta y_{i t-j}+\alpha_{i} d_{t}+e_{i t} .
$$

The LLC test can be implemented in three steps. The first step is to determine the lag order $P_{i}$. This is done by first estimating (2) for each individual without imposing a common AR parameter. The appropriate lag order can then be determined preferably using a data dependent rule. LLC suggest using the Campbell and Perron (1991) rule, which is a simple sequential test rule based on the significance of the individual lag parameters $\phi_{i j}$. Another possibility is to use an information criterion, such as the Schwarz Bayesian criterion. Alternatively, the number of lags can be determined independently of the data. Two examples of such deterministic rules involves choosing $P_{i}$ arbitrarily or as a fixed function of $T$, which ensures that the estimated test regression provides an increasingly good approximation of the possibly infinite AR process in Assumption 1 (b).

Once the appropriate lag order in (2) has been determined, the following two auxiliary regressions are estimated by least squares

$$
\begin{aligned}
\Delta y_{i t} & =\sum_{j=1}^{P_{i}} \phi_{i j} \Delta y_{i t-j}+\alpha_{i} d_{t}+e_{i t}, \\
y_{i t-1} & =\sum_{j=1}^{P_{i}} \phi_{i j} \Delta y_{i t-j}+\alpha_{i} d_{t}+v_{i t-1} .
\end{aligned}
$$


This yields two sets of orthogonalized residuals, here denoted $\widehat{e}_{i t}$ and $\widehat{v}_{i t-1}$. Also, to control for heterogeneity across the individuals, $\widehat{e}_{i t}$ and $\widehat{v}_{i t-1}$ are further normalized as $\widetilde{e}_{i t}=\widehat{e}_{i t} / \widehat{\sigma}_{i}$ and $\widetilde{v}_{i t-1}=\widehat{v}_{i t-1} / \widehat{\sigma}_{i}$, where $\widehat{\sigma}_{i}^{2}$ is a consistent estimate of $\sigma_{i}^{2}$, which can be obtained as the residual variance from a regression of $\widehat{e}_{i t}$ on $\widehat{v}_{i t-1}$.

The second step in computing the LLC test is to calculate the following variance ratio

$$
\widehat{S}_{N}=\frac{1}{N} \sum_{i=1}^{N} \widehat{\omega}_{i} / \widehat{\sigma}_{i}
$$

where

$$
\widehat{\omega}_{i}^{2}=\frac{1}{T} \sum_{t=1}^{T}\left(\Delta y_{i t}\right)^{2}+\frac{2}{T} \sum_{j=1}^{K_{i}-1}\left(1-\frac{j}{K_{i}}\right) \sum_{t=j+1}^{T} \Delta y_{i t} \Delta y_{i t-j}
$$

The quantity $\widehat{\omega}_{i}^{2}$ is a semiparametric consistent estimator of $\omega_{i}^{2}$ under the null hypothesis. The weight function $1-j / K_{i}$ is the Bartlett kernel, which depends on the bandwidth parameter $K_{i}$. As with $P_{i}$, this parameter may be chosen based on the data or it may be chosen independently of the data. Two of the most popular approaches are to choose $K_{i}$ according to the Newey and West (1994) automatic bandwidth rule or as a fixed function of $T$. The motivation behind the latter is that $\widehat{\omega}_{i}^{2}$ will only be consistent for $\omega_{i}^{2}$ if $K_{i}$ is allowed to grow with $T$. For this to happen, the relative expansion rate of $K_{i}$ must be such that the ratio of $K_{i}$ to $T$ goes to zero.

Note that $\widehat{\omega}_{i}^{2}$ is not based on the same residuals used for computing $\sigma_{i}^{2}$ but rather on the first differences of $y_{i t}$. Although seemingly innocent, as will be explained later, this small modification is actually very important in the sense that if $\widehat{\omega}_{i}^{2}$ is based on residuals instead of differences, the LLC test is no longer consistent. Note also that the above estimation procedure does not account for any deterministic terms, and that it needs to be modified to accommodate the constant and trend in Models 2 and 3, respectively. This requires replacing $\Delta y_{i t}$ in $\widehat{\omega}_{i}^{2}$ by the fitted residuals from a first-stage regression of $\Delta y_{i t}$ onto $d_{t}$, the vector of deterministic components.

In the third step, all observations on $\widetilde{e}_{i t}$ and $\widetilde{v}_{i t-1}$ are pooled, in which case $\delta$ can be estimated from the following regression

$$
\widetilde{e}_{i t}=\delta \widetilde{v}_{i t-1}+\text { error. }
$$

Let $\widetilde{\delta}$ denote the least squares estimate of $\delta$ in (6). If we further let $\widehat{\sigma}^{2}$ denote the estimated error variance from that regression, then the standard error of $\widetilde{\delta}$ is given by

$$
S E(\widetilde{\delta})=\left(\widehat{\sigma}^{-2} \sum_{i=1}^{N} \sum_{t=2}^{T} \widetilde{v}_{i t-1}^{2}\right)^{-1 / 2}
$$


The $t$-ratio for testing $\delta=0$ can now be written as

$$
t_{\delta}=\frac{\widetilde{\delta}}{S E(\widetilde{\delta})}
$$

It is well-known that, while unbiased in Model 1, $\widetilde{\delta}$ is downwards biased in Models 2 and 3 with individual specific intercepts and trends. Thus, without any modification, since $\delta<0$ under the alternative hypothesis, $t_{\delta}$ will tend to reject the null too frequently in small samples. To account for this, LLC propose using the following adjusted test statistic

$$
t_{\delta}^{*}=\frac{t_{\delta}-N T \widehat{S}_{N} \widehat{\sigma}^{-2} S E(\widetilde{\delta}) \mu_{m}^{*}}{\sigma_{m}^{*}} .
$$

The quantities $\mu_{m}^{*}$ and $\sigma_{m}^{*}$ are certain mean and standard deviation adjustment terms that depend on the choice of model as indicated by the subscript $m$. As expected, $\mu_{m}^{*}$ is zero in Model 1 and negative in Models 2 and 3. Numerical values of $\mu_{m}^{*}$ and $\sigma_{m}^{*}$ are provided in Table 2 of LLC. The remaining quantities $\widehat{S}_{N}, \widehat{\sigma}^{2}$ and $S E(\widetilde{\delta})$ are necessary in order to render the asymptotic distribution of $t_{\delta}^{*}$ free of the nuisance parameters induced by permitting for a completely heterogeneous serial correlation structure.

As for the asymptotic null distribution of $t_{\delta}^{*}$, LLC show that, under the Assumptions 1 and 2 , as $T \rightarrow \infty$ and $N \rightarrow \infty$ jointly, then

$$
t_{\delta}^{*} \Rightarrow N(0,1) \text {. }
$$

Moreover, although LLC do not provide any analysis of their test statistic under the alternative hypothesis, we speculate that it is consistent. The argument goes as follows. Suppose that $\delta<0$ so that the alternative is true. Since the data is stationary in this case, $\sqrt{N T}(\widetilde{\delta}-\delta)$ must be $O_{p}(1)$, which implies that

$$
\frac{1}{\sqrt{N T}} t_{\delta}=\frac{\delta}{\sqrt{N T} S E(\widetilde{\delta})}+\frac{1}{\sqrt{N T}} \frac{\sqrt{N T}(\widetilde{\delta}-\delta)}{\sqrt{N T} S E(\widetilde{\delta})}=O_{p}(1)
$$

where we have used the fact that $\sqrt{N T} S E(\widetilde{\delta})$ converges to the asymptotic variance of $\sqrt{N T}(\widetilde{\delta}-\delta)$, so this term must also be $O_{p}(1) .{ }^{2}$ This shows that $t_{\delta}$, the unadjusted test statistic, must be $O_{p}(\sqrt{N T})$ under the alternative.

The asymptotic behavior of the adjusted statistic $t_{\delta}^{*}$ under the alternative depends to a large extent on what happens to the mean adjustment term

$$
N T \widehat{S}_{N} \widehat{\sigma}^{-2} S E(\widetilde{\delta}) \mu_{m}^{*} \text {. }
$$

This is so because although $t_{\delta}$ is diverging towards negative infinity at rate $\sqrt{N T}$, the adjustment term is diverging in the other direction. In other words,

\footnotetext{
${ }^{2}$ As usual, for any real $r$ and random variable $y_{n}$ with $n=1, \ldots, N$, we use the notation $O_{p}\left(N^{r}\right)$ to indicate that $y_{n}$ is at most of order $N^{r}$ in probability, which essentially means that $y_{n} / N^{r}$ remains bounded in the limit as $N$ grows.
} 
the consistency of $t_{\delta}^{*}$ hinges on the adjustment term being of smaller order in probability than $\sqrt{N T}$. Towards this end, note that because $y_{i t}$ is stationary, $\Delta y_{i t}$ is over-differentiated with no variance at zero frequency. Thus, in contrast to what happens under the null, in this case $\widehat{\omega}_{i}^{2}$ does not converge to its true value of $\omega_{i}^{2}$ but in fact vanishes as $T$ and $K_{i}$ grow suggesting that $\widehat{S}_{N}$ vanishes too. If the decay of $\widehat{S}_{N}$ is quick enough, the mean adjustment term should become negligible, in which case $t_{\delta}^{*}$ diverges to negative infinity at the same rate as $t_{\delta}$.

The problem here is that the rate at which $\widehat{\omega}_{i}^{2}$ approaches zero can be slow unless the bandwidth parameter $K_{i}$ is very large. In fact, as will be shown in Section 5, most common rules for determining $K_{i}$ are likely to be hopelessly inadequate. In this case, $\widehat{S}_{N}$ does not vanish and it is perfectly possible that the mean adjustment term will actually increase as $T$ and $N$ grow. Thus, since $\mu_{m}^{*}$ is negative in Models 2 and 3, this affects the test by shifting its distribution to the right, thus causing a loss of power.

To appreciate the issues involved, write

$$
\begin{aligned}
N T \widehat{S}_{N} \widehat{\sigma}^{-2} S E(\widetilde{\delta}) \mu_{m}^{*} & =N T \widehat{S}_{N} \widehat{\sigma}^{-2} \mu_{m}^{*}\left(\widehat{\sigma}^{-2} \sum_{i=1}^{N} \sum_{t=2}^{T} \widetilde{v}_{i t-1}^{2}\right)^{-1 / 2} \\
& =\sqrt{N T} \widehat{S}_{N} \widehat{\sigma}^{-1} \mu_{m}^{*}\left(\frac{1}{N T} \sum_{i=1}^{N} \sum_{t=2}^{T} \widetilde{v}_{i t-1}^{2}\right)^{-1 / 2} \\
& =\sqrt{N T} \widehat{S}_{N} O_{p}(1) .
\end{aligned}
$$

Note that, since $y_{i t}$ is stationary, the term within parentheses is $O_{p}(1)$, and in fact converges to a constant as $N$ and $T$ grow. Of course, this stands in sharp contrast to the unit root case, in which this term is $O_{p}(T)$, and thus diverges with $T$. This distinction is important because it means that the probabilistic order of the mean adjustment term actually increases by a factor $\sqrt{T}$ under the alternative.

To see what effect this has on $t_{\delta}^{*}$, note that the orders of both the mean adjustment and $t_{\delta}$ contain the term $\sqrt{N}$. We can therefore just as well assume a fixed $N$ and concentrate on $T$, in which case the order of the mean adjustment reduces to $\widehat{S}_{N} O_{p}(\sqrt{T})$. Hence, for this term to vanish, each of the long-run variances $\widehat{\omega}_{i}$ in $\widehat{S}_{N}$ must go to zero at a faster rate than $\sqrt{T}$, which requires a very large choice of $K_{i}$. In fact, in the appendix we show that

$$
\widehat{\omega}_{i}=O_{p}\left(\frac{1}{\sqrt{K_{i}}}\right) \text { as } T, K_{i} \rightarrow \infty \text { with } \frac{K_{i}}{T} \rightarrow 0 .
$$

In other words, $\widehat{\omega}_{i}$ disappears with $K_{i}$ at rate $\sqrt{K_{i}}$. This is very interesting because it means that even if we choose $K_{i}$ close to $T$, which is the largest possible choice without violating the requirement that $K_{i} / T$ must go to zero, the adjustment term will not disappear. In this sense, the LLC recommendation 
of setting $K_{i}$ proportional to $T^{1 / 3}$ is not enough. Of course, the test is till diverging but at a slower rate as the mean adjustment term now increases with $T{ }^{3}$ Thus, unless we are considering Model 1 without any deterministic constant or trend terms, in which case $\mu_{m}^{*}$ is zero, we will always loose power by using $t_{\delta}^{*}$ rather than $t_{\delta}$.

\section{A modified test}

One way to circumvent the problem with the LLC proposal is to modify their test so that it does not rely to such a large extent on the behavior of the mean adjustment term. The idea put forth in this section is to modify the test statistic itself rather than the adjustment term.

The modified test that we consider is based on the following $t$-ratio

$$
\tau_{\delta}=\frac{\widehat{\delta}}{S E(\widehat{\delta})},
$$

where $\widehat{\delta}$ is the least squares estimate of the slope in a regression of $\widetilde{e}_{i t}=\widehat{e}_{i t} / \widehat{\sigma}_{i}$ on $\widetilde{v}_{i t-1}=\left(\widehat{v}_{i t-1} \widehat{\phi}_{i}(1)\right) / \widehat{\sigma}_{i}$, where $\widehat{\phi}_{i}(1)$ is any consistent estimate of $\phi_{i}(1)$. The standard error of $\widehat{\delta}$ is defined as

$$
S E(\widehat{\delta})=\left(\sum_{i=1}^{N} \sum_{t=2}^{T} \widetilde{v}_{i t-1}^{2}\right)^{-1 / 2} .
$$

Note that the only two differences between $t_{\delta}$ and $\tau_{\delta}$, its modified version, are the terms $\widehat{\phi}_{i}(1)$ and $\widehat{\sigma}^{2}$ appearing in $\widetilde{v}_{i t-1}$ and $S E(\widetilde{\delta})$, respectively.

As with $t_{\delta}$, although the asymptotic distribution of $\tau_{\delta}$ is normal, it does not have zero mean and unit variance. To account for this, we suggest using the following adjusted statistic

$$
\tau_{\delta}^{+}=\frac{\tau_{\delta}-\sqrt{N} \mu_{m}}{\sigma_{m}} .
$$

It is important to note that in contrast to $t_{\delta}^{*}$, the mean and variance adjustment terms $\mu_{m}$ and $\sigma_{m}^{2}$ in (9) derive directly from the underlying Brownian motion $W_{i}(r)$, and hence do not involve any estimated quantities such as $\widehat{S}_{N}$. This does not only make the modified test computationally very simple, but also eliminates the need for $\widehat{\omega}_{i}^{2}$ to go to zero under the alternative hypothesis. The sequential limit distribution of the modified statistic is given in the next theorem.

Theorem 1. (Asymptotic distribution.) Under Assumptions 1 and 2, and the null hypothesis, as $T \rightarrow \infty$ and then $N \rightarrow \infty$ sequentially

$$
\tau_{\delta}^{+} \Rightarrow N(0,1)
$$

\footnotetext{
${ }^{3}$ Note that if $K_{i}$ is fixed independently of $T$, then $t_{\delta}^{*}$ is no longer consistent, as the mean adjustment term is now diverging at the same rate as $t_{\delta}$.
} 
Remark 1. The proof of Theorem 1 is given in the appendix. The theorem states that the unadjusted statistic $\tau_{\delta}$ converges to a standard normal variate after centering it by $\mu_{m}$ and scaling it by $\sigma_{m}$. As with $\mu_{m}^{*}$ and $\sigma_{m}^{*}$, the exact numerical values of $\mu_{m}$ and $\sigma_{m}$ depend on the deterministic specification of the data generating process. These values can be obtained either by simulation methods or by direct calculation using the moments of Brownian motion. This paper uses the latter approach. The results are summarized in Table 1.

Remark 2. As already mentioned, besides the more robust construction of the mean adjustment term, $\tau_{\delta}$ has the additional operational advantage that it is very simple. In fact, the only complicating factor here is that it requires a consistent estimator of $\phi_{i}(1)$. A vary natural way to do this is to use a parametric approach, and to estimate $\phi_{i}(1)$ using the formula

$$
\widehat{\phi}_{i}(1)=1-\sum_{j=1}^{P_{i}} \widehat{\phi}_{i j},
$$

where $\widehat{\phi}_{i j}$ is the least squares estimate of the lag parameter $\phi_{i j}$ from the augmented regression in (2). Note that, in contrast to LLC, this approach does not involve any semiparametric estimation, which means that $\tau_{\delta}^{+}$can be fully parametric. Alternatively, $\phi_{i}(1)$ can be estimated using the ratio $\widehat{\sigma}_{i} / \widehat{\omega}_{i}$, which again requires semiparametric kernel estimation of the long-run variance $\widehat{\omega}_{i}^{2}$. Even so, it is important to realize that it is no longer necessary for $\widehat{\omega}_{i}^{2}$ to go to zero under the alternative hypothesis, which makes the modified test relatively robust in the sense that it does not depend to such a large extent on how $K_{i}$ is chosen. Moreover, $\widehat{\omega}_{i}^{2}$ does not has to be based on $\Delta y_{i t}$, but can be computed from the same residuals used for obtaining $\widehat{\sigma}_{i}^{2}$, namely those obtained by regressing $\widehat{e}_{i t}$ on $\widehat{v}_{i t-1}$. As pointed out by Breitung (2000), this is not possible when using $t_{\delta}^{*}$, because then $\widehat{\omega}_{i}^{2}$ will no longer vanish under the alternative hypothesis, which makes the LLC test inconsistent.

Remark 3. As indicated in Section 2, it is not the allowance for nonzero intercept and trend terms per se that makes the testing complicated but the allowance for such terms while simultaneously permitting for a fully heterogeneous serial correlation structure. If there is no serial correlation, then the adjusted statistic $\tau_{\delta}^{+}$can be computed with $\tau_{\delta}$ replaced by the conventional $t$-statistic of a zero slope in a pooled least squares regression of $\Delta y_{i t}$ on $d_{t}$ and $y_{i t-1}$, which of course greatly simplifies the computation of the test.

Remark 4. Although $\tau_{\delta}^{+}$has many advantages, it also has two drawbacks. One drawback is that its variance is larger than that of $t_{\delta}^{*}$. To appreciate this, note that from the appendix we have that $\sigma_{m}^{2}$ can be written as $\Theta_{1}^{-1} \Sigma_{22}+$ $\frac{1}{4} \Theta_{2}^{2} \Theta_{1}^{-3} \Sigma_{11}$, where $\Theta$ and $\Sigma$ are the expected value and variance of a certain vector Brownian motion, respectively. It follows that since the square of $\sigma_{m}^{*}$ is identically $\Theta_{1}^{-1} \Sigma_{22}$, and since $\frac{1}{4} \Theta_{2}^{2} \Theta_{1}^{-3} \Sigma_{11}$ is greater than zero in Models 2 and 
$3, \sigma_{m}$ is at least as large as $\sigma_{m}^{*}$. Another drawback is that unlike $\widetilde{\delta}, \widehat{\delta}$ is not a consistent estimator of $\delta$ under the alternative hypothesis. However, note that by using the same arguments as in (8), it is clear that although $\sqrt{N T}(\widehat{\delta}-\delta)$ diverges at rate $\sqrt{N T}, \tau_{\delta}$ is still $O_{p}(\sqrt{N T})$. Thus, the inconsistency of $\widehat{\delta}$ does not matter asymptotically.

\section{Simulation evidence}

In this section, we provide some small-sample evidence on the size and power of $t_{\delta}^{*}$ and $\tau_{\delta}^{+}$. For convenience of comparison, the basic design of the Monte Carlo study is taken from LLC. However, these authors do not consider the effects of serial correlation, an important feature when considering lag and bandwidth selection, and we have therefore extended their design to allow for dependent innovations. The resulting data generating process can be written as

$$
y_{i t}=\rho y_{i t-1}+u_{i t} \text { with } u_{i t}=\phi u_{i t-1}+e_{i t},
$$

where $e_{i t} \sim N(0,1)$. The data is generated for 3,000 panels with $N$ crosssectional and $T+50$ time series observations. The first 50 observations for each $i$ is then disregarded to reduce the effect of the initial conditions of $y_{i t}$ and $e_{i t}$, which are both set to zero.

We have two experiments, one for each test. In the first, we examine the size and power for $t_{\delta}^{*}$ when using different types of rules for determining $P_{i}$ and $K_{i}$. For the selection of the lag length $P_{i}$, we consider three rules. They are the sequential test rule of Campbell and Perron (1991), the Schwarz Bayesian information criterion based rule and the zero lag rule, which was used by LLC in their Monte Carlo study. Consistent with the results of $\mathrm{Ng}$ and Perron (1995), the maximum number of lags for the first two rules is allowed to increase with $T$ at the rate $4(T / 100)^{2 / 9}$. For the bandwidth parameter $K_{i}$, we also have three rules. The first is the data dependent rule first explored by Andrews (1991), and then extended by Newey and West (1994). The remaining two rules are deterministic, and involve setting $K_{i}$ either equal to $4(T / 100)^{2 / 9}$ as suggested by Newey and West (1994) or equal to $3.21 T^{1 / 3}$ as in LLC.

Both the parametric and semiparametric versions of $\tau_{\delta}^{+}$are simulated. For the latter, we follow the recommendation of Phillips and Perron (1988), and construct $\widehat{\omega}_{i}^{2}$ using residuals instead of first differences. The LLC test can be constructed in two ways depending on the choice of mean and variance adjustment, which can be either asymptotic or small-sample specific. LLC tabulate both types. The asymptotic adjustments are best in the sense that they facilitate a more straightforward comparison with $\tau_{\delta}^{+}$, which is based on the asymptotic adjustments provided in Table 1 . On the other hand, the small-sample adjustments are probably more likely to be used in practice, and we therefore also present some results for the small-sample adjusted LLC test. 
For brevity, we only report the size and power on the $5 \%$ significance level. Since size accuracy is not perfect, all powers are adjusted so that each test has the same level of $5 \%$ when the null hypothesis is true. ${ }^{4}$ All computational work has been performed in GAUSS.

\subsection{The LLC test}

In the first experiment, we examine the performance of the $t_{\delta}^{*}$ test. The purpose here is to show that the simulation results presented by LLC depend to a large extent on their choice of lag length and bandwidth, and that the use of alternative selection rules is likely to result in deceptive inference.

Consider first the results of the size for the $t_{\delta}^{*}$ test, which are presented in Table 2. As seen from the table, the performance is rather mixed. If there is no serial correlation, or the test is performed in Model 1 with no deterministic components, then the nominal level is generally maintained quite well and there are only small distortions. On the other hand, if we look at Models 2 and 3, we find that the test can be quite distorted, even when $\phi$ is zero and there is no serial correlation. As expected, these distortions are greatly exacerbated when $\phi$ is nonzero. We also see that the distortions have a tendency to accumulate and to become even more serious as $N$ increases.

As for the different lag and bandwidth selection rules, we find that the test based on the LLC specification with $K_{i}=3.21 T^{1 / 3}$ and $P_{i}$ set to zero generally suffers from the most massive distortions, especially in Model 3 with deterministic trends. This is not totally unexpected, however, given that the asymptotic adjustment terms tend to lie quite far from their small-sample counterparts obtained from the simulated data. This is reflected in the results based on the small-sample adjustments, which are much less distorted.

Unfortunately, these small-sample adjustments are only valid for the special case when $K_{i}=3.21 T^{1 / 3}$ and $P_{i}$ equals zero, which severely limits their applicability. Indeed, unreported simulation results suggest that when these adjustments are used in combination with other lag or bandwidth rules, $t_{\delta}^{*}$ generally becomes severely distorted. Another drawback is that these adjustments have been generated while disregarding the effects of serial correlation. This is clearly visible from Table 2, which shows that the small-sample adjusted LLC test is just as distorted as its asymptotic counterpart when $\phi$ is nonzero.

Consider next the results of the power of the $t_{\delta}^{*}$ test, which are presented in Table 3. In this case, we set $\phi$ to zero and focus on the performance for different local alternatives, as indicated by the AR parameter $\rho$. The first thing to note is the overall good performance of the test based on the LLC specification of

\footnotetext{
${ }^{4}$ In addition to the results reported here, we have experimented with a large number of different parameterizations of the data generating process, including heterogeneous AR errors, heterogeneous and homogenous moving average errors, and nonzero deterministic intercept and trend terms. Except possibly for the usual distortions in the case with large negative moving average errors, the conclusions were not altered.
} 
$P_{i}$ and $K_{i}$. However, we also see that when $K_{i}$ is chosen differently, then the power can be extremely poor, and practically nonexisting in most experiments.

Of course, this finding is well in line with the argument that $t_{\delta}^{*}$ should suffer from low power unless $K_{i}$ is very large. Moreover, since adding more lags affects the test by increasing the number of parameters that needs to be estimated, choosing $P_{i}$ greater than zero is expected to reduce the power of the test even further. This effect is also clearly visible in the table. Similarly, the power decreases as the number of deterministic components increases.

The results look much better in Model 1, for which Table 3 suggests that $t_{\delta}^{*}$ has good power in all experiments considered. The reason for this drastic improvement in comparison to Models 2 and 3 is of course that $\mu_{m}^{*}$ equals zero in Model 1, which implies that the power of the test is unaffected by the behavior of the mean adjustment term.

\subsection{The modified test}

Next, we continue to the results on the second experiment contained in Tables 4 trough 6 , where the size and power of the $\tau_{\delta}^{+}$test is evaluated. Tables 4 and 5 summarize the results for the test based on the semiparametric estimator of $\phi_{i}(1)$, while Table 6 contains the results for the test based on the parametric estimator.

Similar to $t_{\delta}^{*}$, Table 4 reveal that $\tau_{\delta}^{+}$generally performs well with near perfect size accuracy in Model 1 but that there is a tendency to overreject in Models 2 and 3, especially when the most generous bandwidth rule $3.21 T^{1 / 3}$ is used. However, when compared to the results reported for $t_{\delta}^{*}$ in Table 2, the distortions of the $\tau_{\delta}^{+}$test are not very large. Indeed, as long as $P_{i}$ and $K_{i}$ are not chosen as in LLC, with 10 cross-sectional units size accuracy is actually quite reasonable. The results reported in Table 6 for the parametric test are even better. In fact, given that $P_{i}$ is larger than zero, size accuracy is almost perfect, even in the smallest panel with 50 time series and 10 cross-sectional units.

As for the relative performance of the tests under the alternative hypothesis of stationarity, Tables 3, 5 and 6 reveal that there are massive power advantages to the $\tau_{\delta}^{+}$test. In fact, based on the results presented here, $\tau_{\delta}^{+}$is uniformly more powerful than $t_{\delta}^{*}$. In addition, while there is clear positive relationship between sample size and power for $\tau_{\delta}^{+}$, the results presented in Table 3 suggest that this is not necessarily the case for $t_{\delta}^{*}$. Thus, the power advantage to the modified test is expected to grow with the sample size. Among the two versions of $\tau_{\delta}^{+}$, the results indicate that while the semiparametric test tend to be more powerful, the gain in power relative to the parametric test is generally very small.

In summary, we find that the modified test show small size distortions and, at the same time, maintain good power in small samples. The LLC test, on the other hand, show large size distortions and, in most cases, no power beyond its size. This finding suggests that the modified test should be a useful addition to the existing menu of panel unit root tests. We also find that, among the 
two modifications considered, the fully parametric test generally performs best. Thus, since this test is also the simplest one to implement, it should be well suited for applied work.

\section{An empirical illustration}

Over the years there has been many studies trying to determine the persistence of inflation, which has been viewed as an important topic because inflation is typically regarded as a key variable in many economic models, whose validity hinges critically on whether inflation is stationary or not. One such study is that of Cluver and Papell (1997), in which the authors deduce evidence based on the LLC test suggesting that inflation is stationary. ${ }^{5}$

The purpose of this section is to simply illustrate the potential effects of using the LLC test in combination with a data dependent choice of lag length and bandwidth. The data that we use are monthly and are taken directly from Culver and Papell (1997). It covers 13 OECD countries between February 1957 and September 1994, which means that there are a total of 5,902 observations available. ${ }^{6}$ As in Culver and Papell (1997), we consider three subpanels, the full panel, the G7 panel and the non-G7 panel.

All tests are constructed exactly as described in Section 5. The empirical results are presented in Table 7 . In agreement with our simulation results, we see that the $t_{\delta}^{*}$ test is unable to reject the unit root null on all conventional significance levels unless the most generous $3.21 T^{1 / 3}$ rule is used. In fact, most test values are positive, and thus not even in the left tail of the distribution. Given this variation in test outcome, whatever the conclusion, it is deemed to be highly unreliable. By contrast, when using the $\tau_{\delta}^{+}$test, we are always able to safely reject the null, which is also consistent with what might have been expected based on the simulations results, and the fact that inflation is usually thought of as being stationary in the long term.

These results clearly illustrates the hazards involved in using the LLC test in empirical work. The modified test is much more robust, and is therefore expected to result in better and more accurate conclusions.

\section{Conclusions}

In this paper, we point out a weakness in the LLC panel unit root test. The problem lies in the adjustment term required to account for the nonzero mean of the test statistic in the presence of deterministic intercept and trend terms.

\footnotetext{
${ }^{5}$ The test used by Cluver and Papell (1997) is actually a somewhat different from the published LCC test, which builds on earlier work by the two first authors. However, in this section, we do not bother with the distinction.

${ }^{6}$ The data is downloadable from Journal of Applied Econometrics data archive available online at http://qed.econ.queensu.ca/jae/.
} 
Specifically, in order to ensure that the test is consistent, it is necessary that this term does not grow too fast with $T$ when evaluated under the stationary alternative. If the growth rate of the adjustment term is sufficiently slow, the statistic diverges to negative infinity suggesting that the test is indeed consistent.

However, the mean adjustment is complicated by the fact that the serial correlation properties of the data is permitted to vary over the cross-section, which makes it dependent on various nuisance parameters. The approach taken by LLC involves correcting the mean adjustment term directly by using both parametric autoregressive lag modelling and semiparametric kernel estimation of these nuisance parameters.

In this paper, we argue that the growth rate of the LLC mean adjustment term is likely to be too fast in small samples unless the kernel bandwidth parameter is chosen very large. In particular, we argue that most known bandwidth selection rules, such as the Andrews (1991) and Newey and West (1994) rules commonly encountered in empirical work, are likely to be hopelessly inadequate, thus leading to a test with low power. Moreover, since adding more lags effectively reduces the number of usable observations, power is expected to be even lower when the test is based on a conservative, large autoregression, modelling approach.

Consistent with this assertion, our simulation results indicate that both the size and power properties of the LLC test can be extremely poor unless the lag length and bandwidth parameters are chosen exactly as in LLC. To mitigate the dependence on the choice of bandwidth, we propose a modified test that relies on correcting the statistic itself rather than the mean adjustment term. This test does not suffer from the same drawbacks as the LLC test, and it is shown to have very good size and power in small samples. We also show that the test can be made fully parametric, which makes it computationally very simple without scarifying small-sample performance. These findings are then verified empirically using as an example the rate of inflation. 


\section{Appendix: Mathematical proofs}

In this appendix, we first analyze the limiting behavior of $\widehat{\omega}_{i}^{2}$, the long-run variance of $\Delta y_{i t}$, and then we proceed to derive the asymptotic null distribution of $\tau_{\delta}^{+}$in Model 1 when the lag order $P_{i}$ is finite. Although $P_{i}$ infinite does not alter the results, the proof needs to be modified and the reader is referred to Said and Dickey (1984) for details. The results for Model 2 follow by simply replacing the Brownian motion $W_{i}(r)$ with its demeaned counterpart $W_{i}(r)-\int_{0}^{1} W_{i}(s) \mathrm{d} s$. Similarly, to obtain the results for Model $3, W_{i}(r)$ is replaced with its demeaned and detrended counterpart $W_{i}(r)+(6 r-4) \int_{0}^{1} W_{i}(s) \mathrm{d} s+(6-12 r) \int_{0}^{1} s W_{i}(s) \mathrm{d} s$.

Lemma A.1. (The probabilistic order of the long-run variance of $\Delta y_{i t}$.) Under Assumptions 1 and 2, and the alternative hypothesis, as $T, K_{i} \rightarrow \infty$ with $K_{i} / T \rightarrow 0$, then $K_{i} \widehat{\omega}_{i}^{2}=O_{p}(1)$.

\section{Proof of Lemma A.1}

We have

$$
\begin{aligned}
\widehat{\omega}_{i}^{2} & =T^{-1} \sum_{t=1}^{T}\left(\Delta y_{i t}\right)^{2}+2 T^{-1} \sum_{j=1}^{K_{i}-1}\left(1-j K_{i}^{-1}\right) \sum_{t=j+1}^{T} \Delta y_{i t} \Delta y_{i t-j} \\
& =T^{-1} \sum_{t=1}^{T}\left(y_{i t}-y_{i t-1}\right)^{2} \\
& +2 T^{-1} \sum_{j=1}^{K_{i}-1}\left(1-j K_{i}^{-1}\right) \sum_{t=j+1}^{T}\left(y_{i t}-y_{i t-1}\right)\left(y_{i t}-y_{i t-j-1}\right) \\
& =T^{-1} \sum_{t=1}^{T}\left(y_{i t}^{2}-2 y_{i t} y_{i t-1}+y_{i t-1}^{2}\right) \\
& +2 \sum_{j=1}^{K_{i}-1}\left(1-j K_{i}^{-1}\right)\left(\widehat{\gamma}_{i j}-\widehat{\gamma}_{i j-1}+T^{-1} y_{i t-1}\left(y_{i t-j-1}-y_{i t-j}\right)\right),
\end{aligned}
$$

where $\widehat{\gamma}_{i j}$ is the estimated $j^{\text {th }}$ order autocovariance of $y_{i t}$. If we assume that $y_{i 0}=0$, then by direct calculation we get

$$
\begin{aligned}
T^{-1} \sum_{t=j+1}^{T} y_{i t-1}\left(y_{i t-j-1}-y_{i t-j}\right) & =\widehat{\gamma}_{i j}+T^{-1} y_{i T}\left(y_{i T-j+1}-y_{i T-j}\right) \\
& -\widehat{\gamma}_{i j+1}-T^{-1} y_{i 1}^{2}
\end{aligned}
$$


which implies that (A1) can be written as

$$
\begin{aligned}
\widehat{\omega}_{i}^{2} & =2 T^{-1} \sum_{t=1}^{T}\left(y_{i t}^{2}-y_{i t} y_{i t-1}\right)+T^{-1} y_{i T}^{2}-2 T^{-1}\left(1-j K_{i}^{-1}\right) y_{i 1}^{2} \\
& +2 \sum_{j=1}^{K_{i}-1}\left(1-j K_{i}^{-1}\right)\left(2 \widehat{\gamma}_{i j}-\widehat{\gamma}_{i j-1}-\widehat{\gamma}_{i j+1}+T^{-1} y_{i T}\left(y_{i T-j-1}-y_{i T-j}\right)\right) .
\end{aligned}
$$

By collecting the autocovatiances appearing in the last term, this expression reduces to

$$
\begin{aligned}
\widehat{\omega}_{i}^{2} & =T^{-1} y_{i T}^{2}-2 T^{-1}\left(1-j K_{i}^{-1}\right) y_{i 1}^{2}+2 K_{i}^{-1}\left(\widehat{\gamma}_{i 0}-\widehat{\gamma}_{i K_{i}}\right) \\
& +2 \sum_{j=1}^{K_{i}-1}\left(1-j K_{i}^{-1}\right) T^{-1} y_{i T}\left(y_{i T-j-1}-y_{i T-j}\right) \\
& =T^{-1} y_{i T}^{2}-2 T^{-1}\left(1-j K_{i}^{-1}\right) y_{i 1}^{2}+2 K_{i}^{-1}\left(\widehat{\gamma}_{i 0}-\widehat{\gamma}_{i K_{i}}\right) \\
& +2 T^{-1}\left(1-j K_{i}^{-1}\right) y_{i T}^{2}-2\left(T K_{i}\right)^{-1} y_{i T}\left(\sum_{j=1}^{K_{i}-1} y_{i T-j}\right) \\
& =T^{-1}\left(3 y_{i T}^{2}-2 y_{i 1}^{2}\right)-2\left(T K_{i}\right)^{-1}\left(y_{i T}^{2}-y_{i 1}^{2}\right)+2 K_{i}^{-1}\left(\widehat{\gamma}_{i 0}-\widehat{\gamma}_{i K_{i}}\right) \\
& -2\left(T K_{i}\right)^{-1} y_{i T}\left(\sum_{j=1}^{K_{i}-1} y_{i T-j}\right)
\end{aligned}
$$

Thus, taking the limit as $T, K_{i} \rightarrow \infty$ with $K_{i} / T \rightarrow 0$, we get

$$
\begin{aligned}
K_{i} \widehat{\omega}_{i}^{2} & =2\left(\widehat{\gamma}_{i 0}-\widehat{\gamma}_{i K_{i}}\right)+K_{i} T^{-1}\left(3 y_{i T}^{2}-2 y_{i 1}^{2}\right)-2 T^{-1}\left(y_{i T}^{2}-y_{i 1}^{2}\right) \\
& -2 T^{-1} y_{i T}\left(\sum_{j=1}^{K_{i}-1} y_{i T-j}\right) \rightarrow p 2 \gamma_{i 0}
\end{aligned}
$$

where $\gamma_{i j}$ is the $j^{\text {th }}$ order autocovariance of $y_{i t}$ and the symbol $\rightarrow_{p}$ indicates convergence in probability. This completes the proof.

Lemma A.2. (Preliminaries for Theorem 1.) Under Assumptions 1 and 2, and the null hypothesis, as $T \rightarrow \infty$
(a) $T^{-1 / 2} y_{i t} \Rightarrow \frac{1}{\phi_{i}(1)} \sigma_{i} W_{i}(r)$;
(b) $T^{-2} \sum_{t=2}^{T} \widehat{v}_{i t-1}^{2} \Rightarrow \frac{1}{\phi_{i}(1)^{2}} \sigma_{i}^{2} \int_{0}^{1} W_{i}(r)^{2} \mathrm{~d} r$;
(c) $T^{-1} \sum_{t=2}^{T} \widehat{v}_{i t-1} \widehat{e}_{i t} \Rightarrow \frac{1}{\phi_{i}(1)} \sigma_{i}^{2} \int_{0}^{1} U_{i}(r) \mathrm{d} W_{i}(r)$. 


\section{Proof of Lemma A.2}

Consider (a). By using the Beveridge-Nelson (BN) decomposition of $\phi_{i}(L)$ as $\phi_{i}(L)=\phi_{i}(1)+\phi_{i}^{*}(L)(1-L)$, the disturbance $u_{i t}$ can be written as

$$
\phi_{i}(L) u_{i t}=\phi_{i}(1) u_{i t}+\phi_{i}^{*}(L) \Delta u_{i t}=e_{i t},
$$

which can be rewritten as

$$
u_{i t}=-\frac{\phi_{i}^{*}(L)}{\phi_{i}(1)} \Delta u_{i t}+\frac{1}{\phi_{i}(1)} e_{i t} .
$$

Now, under the null, Model 1 reduces to $y_{i t}=\sum_{j=1}^{t} u_{i j}$, which implies (a) as can be seen by writing

$$
\begin{aligned}
T^{-1 / 2} \sum_{j=1}^{t} u_{i j} & =-\frac{\phi_{i}^{*}(L)}{\phi_{i}(1)} T^{-1 / 2} \Delta u_{i t}+\frac{1}{\phi_{i}(1)} T^{-1 / 2} \sum_{j=1}^{t} e_{i j} \\
& =\frac{1}{\phi_{i}(1)} T^{-1 / 2} \sum_{j=1}^{t} e_{i j}+o_{p}(1) \\
& \Rightarrow \frac{1}{\phi_{i}(1)} \sigma_{i} W_{i}(r) .
\end{aligned}
$$

Next, consider (b). Let $w_{i t}=\left(\Delta y_{i t-1}, \ldots, \Delta y_{i t-p_{i}}\right)^{\prime}$. By using the rules for projections, $\sum_{t=2}^{T} \widehat{v}_{i t-1}^{2}$ can be written as

$$
\sum_{t=2}^{T} \widehat{v}_{i t-1}^{2}=\sum_{t=2}^{T} y_{i t-1}^{2}-\sum_{t=2}^{T} y_{i t-1} w_{i t}^{\prime}\left(\sum_{t=2}^{T} w_{i t} w_{i t}^{\prime}\right)^{-1} \sum_{t=2}^{T} w_{i t} y_{i t-1} .
$$

By Lemma 2.1 of Park and Phillips (1989), we have that $\sum_{t=2}^{T} y_{i t-1} w_{i t}^{\prime}=O_{p}(T)$ and $\sum_{t=2}^{T} w_{i t} w_{i t}^{\prime}=O_{p}(T)$, which implies

$$
\begin{aligned}
\sum_{t=2}^{T} \widehat{v}_{i t-1}^{2} & =\sum_{t=2}^{T} y_{i t-1}^{2}+O_{p}(T) O_{p}\left(T^{-1}\right) O_{p}(T) \\
& =\sum_{t=2}^{T} y_{i t-1}^{2}+O_{p}(T) .
\end{aligned}
$$

Therefore, by using (a), we obtain the following limit as $T \rightarrow \infty$

$$
T^{-2} \sum_{t=2}^{T} \widehat{v}_{i t-1}^{2}=T^{-2} \sum_{t=2}^{T} y_{i t-1}^{2}+o_{p}(1) \Rightarrow \frac{1}{\phi_{i}(1)^{2}} \sigma_{i}^{2} \int_{0}^{1} W_{i}(r)^{2} \mathrm{~d} r,
$$

which establishes (b). 
Finally, consider (c). We have

$$
\sum_{t=2}^{T} \widehat{v}_{i t-1} \widehat{e}_{i t}=\sum_{t=2}^{T} y_{i t-1} e_{i t}-\sum_{t=2}^{T} y_{i t-1} w_{i t}^{\prime}\left(\sum_{t=2}^{T} w_{i t} w_{i t}^{\prime}\right)^{-1} \sum_{t=2}^{T} w_{i t} e_{i t} .
$$

By using the same arguments as before, we get

$$
\begin{aligned}
\sum_{t=2}^{T} \widehat{v}_{i t-1} \widehat{e}_{i t} & =\sum_{t=2}^{T} y_{i t-1} e_{i t}+O_{p}(T) O_{p}\left(T^{-1}\right) O_{p}\left(T^{1 / 2}\right) \\
& =\sum_{t=2}^{T} y_{i t-1} e_{i t}+O_{p}\left(T^{1 / 2}\right) .
\end{aligned}
$$

The limit of this expression as $T \rightarrow \infty$ is given by

$$
\begin{aligned}
T^{-1} \sum_{t=2}^{T} \widehat{v}_{i t-1} \widehat{e}_{i t} & =T^{-1} \sum_{t=2}^{T} y_{i t-1} e_{i t}+o_{p}(1) \\
& \Rightarrow \frac{1}{\phi_{i}(1)} \sigma_{i}^{2} \int_{0}^{1} W_{i}(r) \mathrm{d} W_{i}(r) .
\end{aligned}
$$

This proves (c).

\section{Proof of Theorem 1}

Define $E_{1 i}=T^{-2} \sum_{t=2}^{T} \widetilde{v}_{i t-1}^{2}, E_{2 i}=T^{-1} \sum_{t=2}^{T} \widetilde{v}_{i t-1} \widetilde{e}_{i t}, E_{1}=\sum_{i=1}^{N} E_{1 i}$ and $E_{2}=\sum_{i=1}^{N} E_{2 i}$, then $\widehat{\delta}$ may be written as

$$
\widehat{\delta}=\left(\sum_{i=1}^{N} \sum_{t=2}^{T} \widetilde{v}_{i t-1}^{2}\right)^{-1} \sum_{i=1}^{N} \sum_{t=2}^{T} \widetilde{v}_{i t-1} \widetilde{e}_{i t}=E_{1}^{-1} E_{2},
$$

which implies that $\tau_{\delta}$ can be rewritten as

$$
\tau_{\delta}=\frac{\widehat{\delta}}{S E(\widehat{\delta})}=E_{1}^{-1 / 2} E_{2}
$$

In order to infer the sequential limit of this statistic, we expand it as

$$
\begin{aligned}
\tau_{\delta}-\sqrt{N} \Theta_{2} \Theta_{1}^{-1 / 2} & =\sqrt{N}\left(N^{-1} E_{2}-\Theta_{2}\right)\left(N^{-1} E_{1}\right)^{-1 / 2} \\
& -\Theta_{2} \sqrt{N}\left(\left(N^{-1} E_{1}\right)^{-1 / 2}-\Theta_{1}^{-1 / 2}\right),
\end{aligned}
$$

where $\Theta$ denote the expectation of the following vector

$$
C_{i}=\left(\int_{0}^{1} W_{i}(r)^{2} \mathrm{~d} r, \int_{0}^{1} W_{i}(r) \mathrm{d} W_{i}(r)\right)^{\prime} .
$$


By using (A4), Lemma A.2 and a law of large numbers (LLN), we have that $N^{-1} E_{1} \rightarrow p \Theta_{1}$ and $N^{-1} E_{2} \rightarrow p \Theta_{2}$ as $T \rightarrow \infty$ prior to $N$. In addition, if we assume that the variance, $\Sigma$ say, of the Brownian motion vector $C_{i}$ is finite, then $\sqrt{N}\left(N^{-1} E_{2}-\Theta_{2}\right) \Rightarrow N\left(0, \Sigma_{22}\right)$ by the Lindberg-Lévy CLT. It follows that the first term on the right hand side of (A4) converges to

$$
\sqrt{N}\left(N^{-1} E_{2}-\Theta_{2}\right)\left(N^{-1} E_{1}\right)^{-1 / 2} \Rightarrow N\left(0, \Theta_{1}^{-1} \Sigma_{22}\right) .
$$

To evaluate the second term, we use the Delta method, which implies that

$$
\sqrt{N}\left(\left(N^{-1} E_{1}\right)^{-1 / 2}-\Theta_{1}^{-1 / 2}\right) \Rightarrow N\left(0, \frac{1}{4} \Theta_{2}^{2} \Theta_{1}^{-3} \Sigma_{11}\right)
$$

These results, together with the fact that $\Sigma$ is block-diagonal, imply that the sequential limit of (A4) can be written as

$$
\tau_{\delta}-\sqrt{N} \Theta_{2} \Theta_{1}^{-1 / 2} \Rightarrow \Theta_{1}^{-1 / 2} N\left(0, \Sigma_{22}\right)-\frac{1}{2} \Theta_{2} \Theta_{1}^{-3 / 2} N\left(0, \Sigma_{11}\right)
$$

Hence, $\tau_{\delta}-\sqrt{N} \Theta_{2} \Theta_{1}^{-1 / 2}$ has zero mean and variance $\Theta_{1}^{-1} \Sigma_{22}+\frac{1}{4} \Theta_{2}^{2} \Theta_{1}^{-3} \Sigma_{11}$, which completes the proof. 
Table 1: Mean and variance adjustment terms.

\begin{tabular}{crr}
\hline \hline Model & \multicolumn{1}{c}{$\mu_{m}$} & \multicolumn{1}{c}{$\sigma_{m}^{2}$} \\
\hline 1 & 0 & 1 \\
2 & $-\sqrt{3 / 2}$ & $8 / 10$ \\
3 & $-\sqrt{15 / 4}$ & $277 / 448$ \\
\hline \hline
\end{tabular}

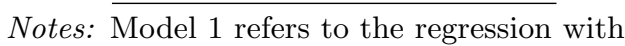
no intercepts or trends, Model 2 refers to the regression with intercept and Model 3 refers to the regression with both intercepts and trends. 


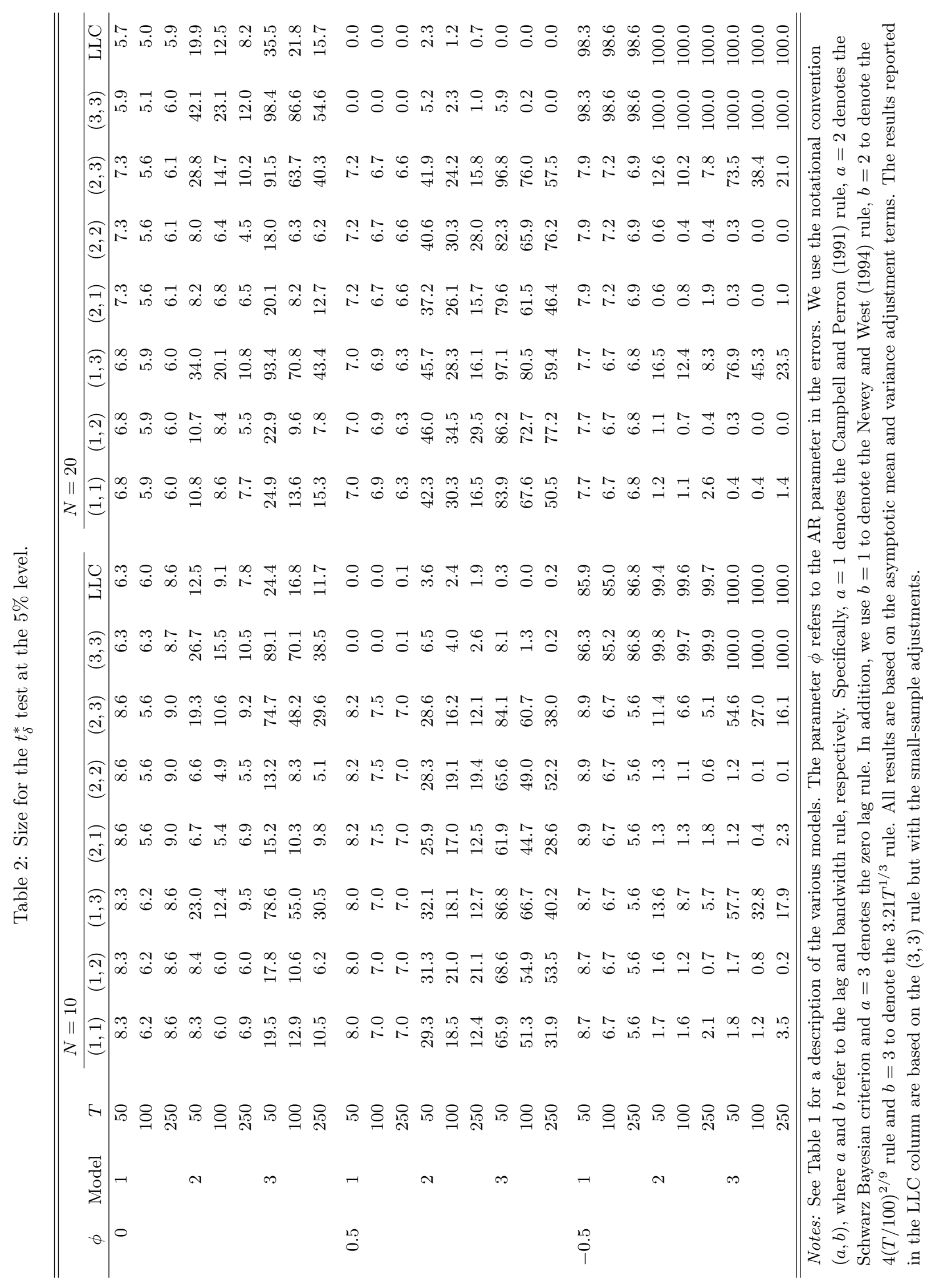




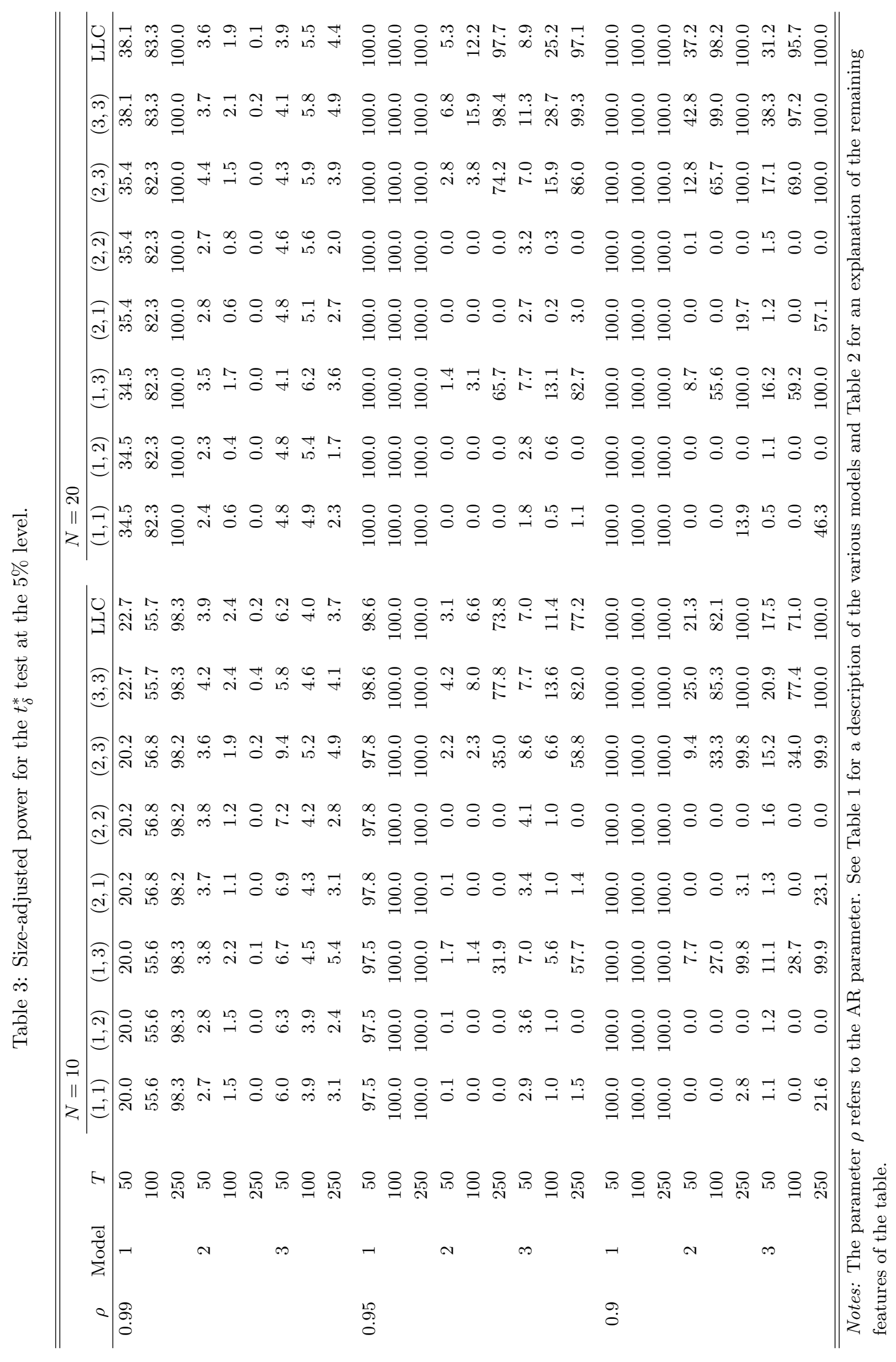




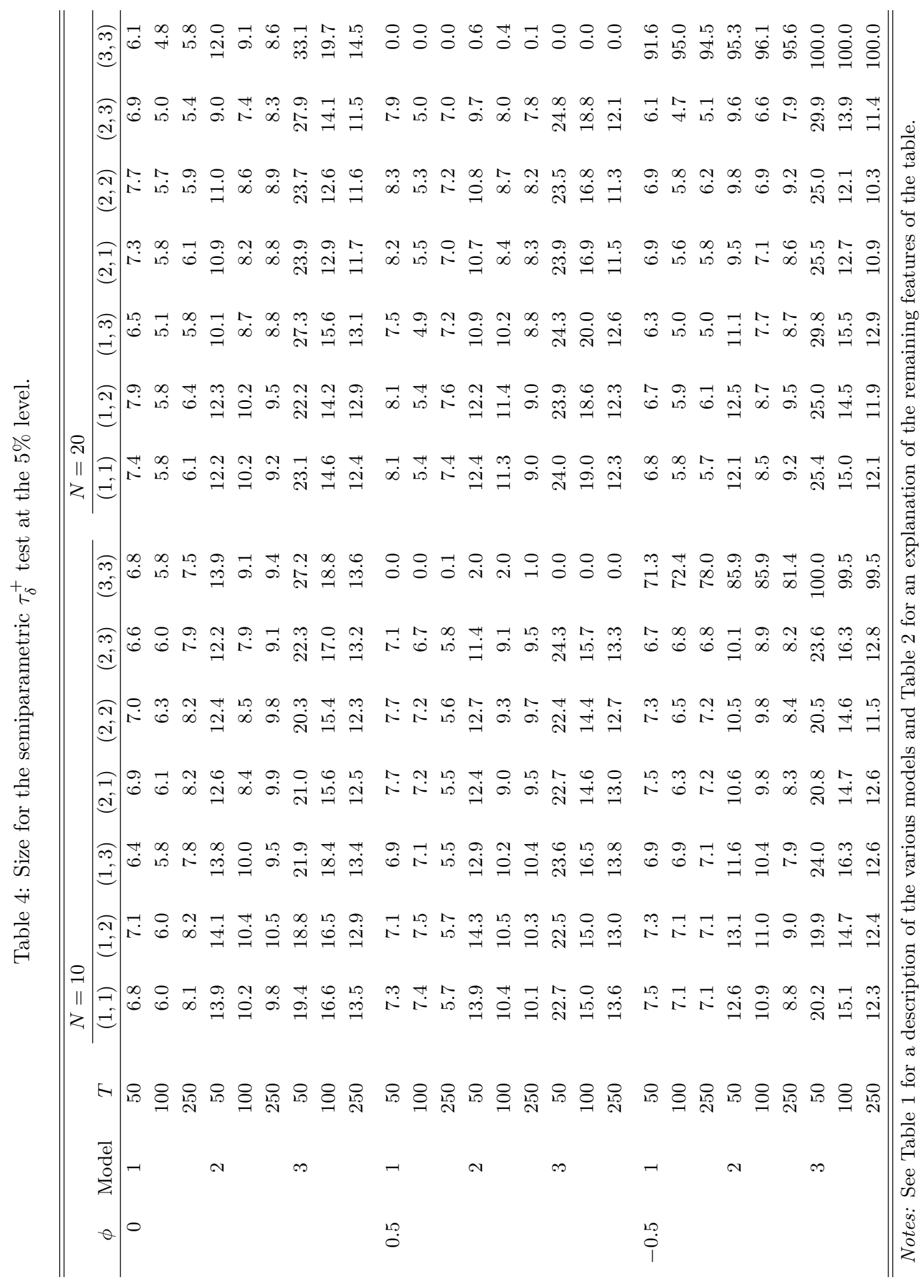




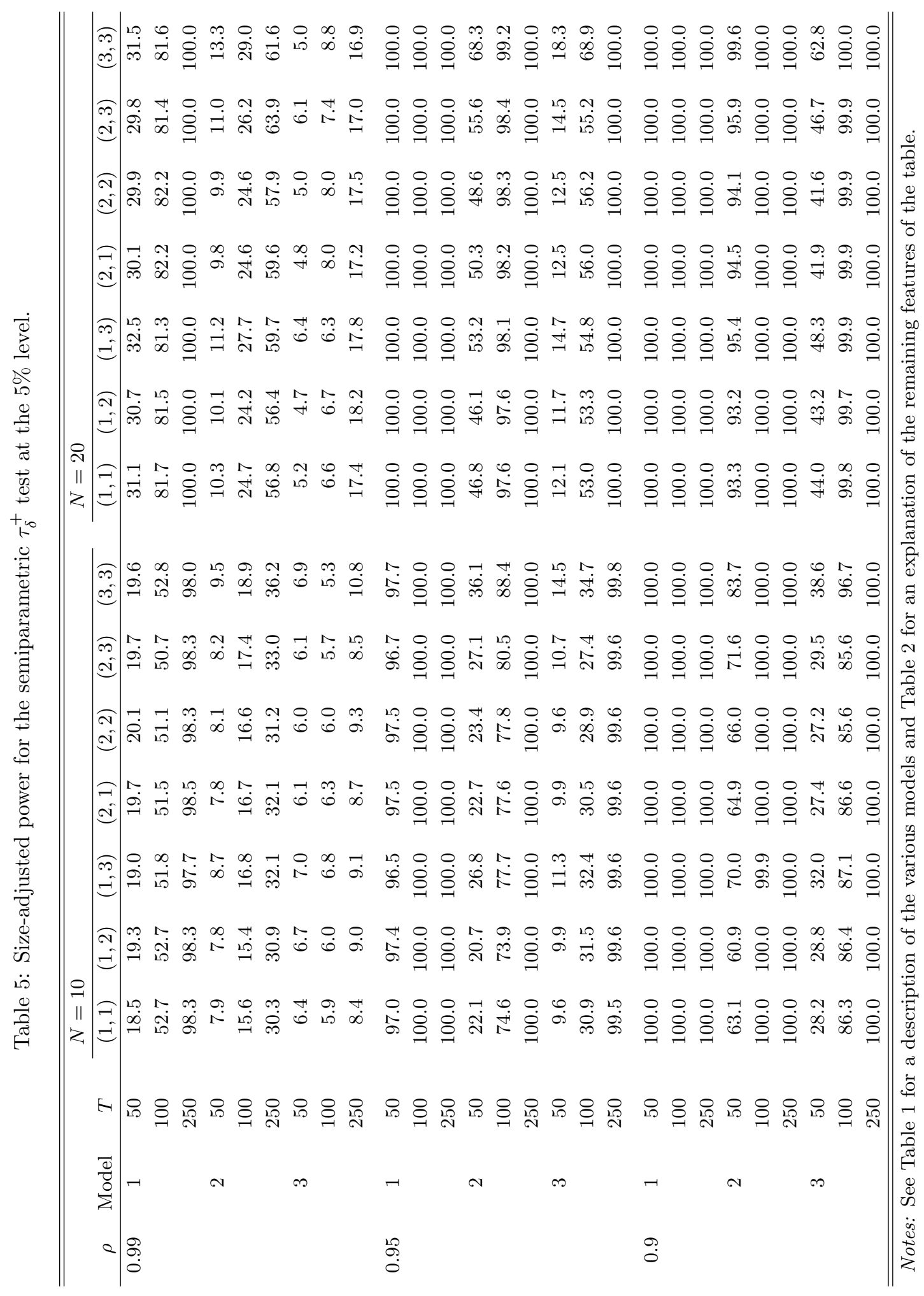




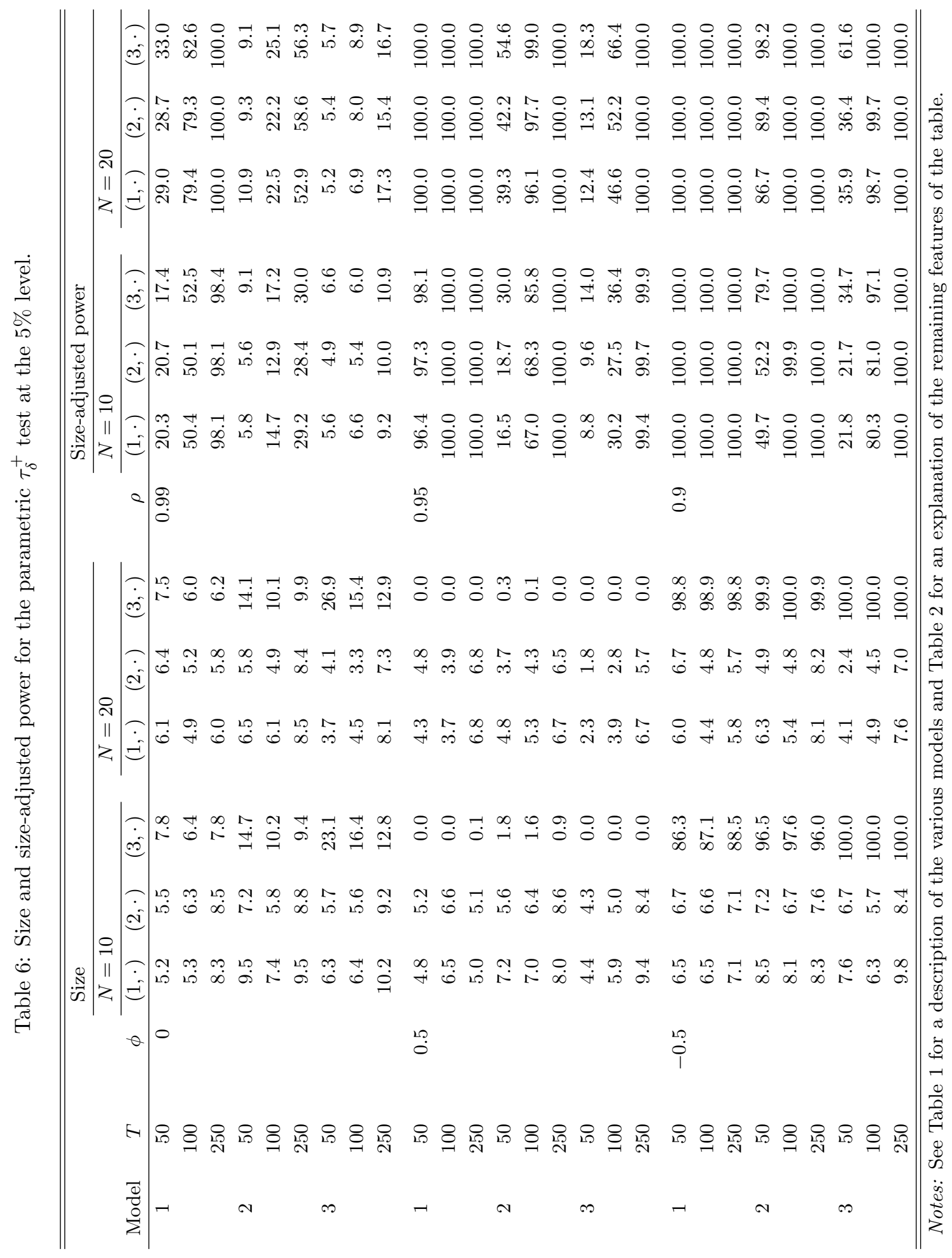




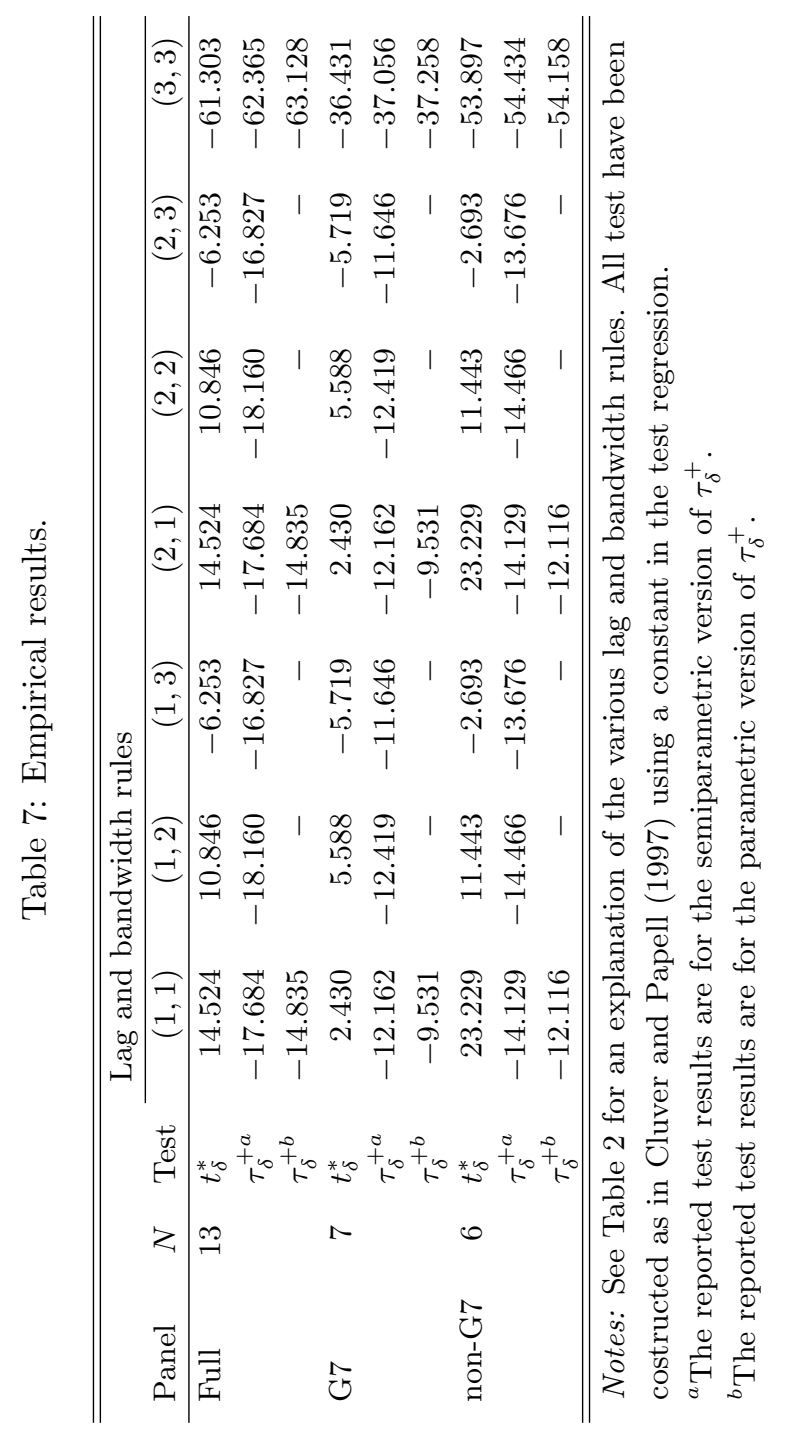




\section{References}

Andrews, D. W. K. (1991). Heteroskedasticity and Autocorrelation Consistent Covariance Matrix Estimation. Econometrica, Vol. 59, pp. 817-858.

Basher, S. A., and M. Mohsin (2004). PPP Tests in Cointegrated Panels: Evidence from Asian Developing Countries. Applied Economics Letters, Vol. 11, pp. 163-166.

Breitung, J. (2000). The Local Power of Some Unit Root Tests for Panel Data. In Baltagi, B. (Ed.), Advances in Econometrics: Nonstationary Panels, Panel Cointegration, and Dynamic Panels, Vol. 15, pp. 161-178, JAI, Amsterdam.

Breitung, J., and S. Das (2005). Panel Unit Root Test Under Cross Sectional Dependence. Statistica Neerlandica, Vol. 59, pp. 414-433.

Campbell, J., and P. Perron (1991). Pitfalls and Opportunities: What Macroeconomists should Know about Unit Roots. In Blanchard, O., and S. Fishers (Eds.), NBER Macroeconomics Annual, MIT Press, Cambridge, MA.

Culver, S. E., and D. H. Papell (1997). Is there a unit root in the inflation rate? Evidence from sequential break and panel data models. Journal of Applied Econometrics, Vol. 12, pp. 436-44.

Crespo-Cuaresma, J., J. Fidrmuc and R. MacDonald (2005). The Monetary Approach to Exchange Rates in the CEECs. Economics of Transition, Vol. 13, pp. 395-416.

Gutierrez, L., and M. M. Gutierrez. (2003). International R\&D Spillovers and Productivity Growth in the Agricultural Sector. A Panel Cointegration Approach. European Review of Agricultural Economics, Vol. 30, pp. 281303.

Hlouskova, J., and M. Wagner (2006). The Performance of Panel Unit Root and Stationarity Tests: Results From a Large Scale Simulation Study. Econometric Reviews, Vol. 25, pp. 85-116.

Jenkins, M. A., and S. M. Snaith (2005). Tests of Purchasing Power Parity via Cointegration Analysis of Heterogeneous Panels with Consumer Price Indices. Journal of Macroeconomics, Vol. 27, pp. 345-362.

Jönsson, K. (2005). Cross-Sectional Dependency and Size Distortion in a Small-Sample Homogenous Panel Data Unit Root Test. Oxford Bulletin of Economics and Statistics, Vol. 67, pp. 369-392.

Kao, C. (1999). Spurious Regression and Residual-Based Tests for Cointegration in Panel Data. Journal of Econometrics, Vol. 90, pp. 1-44. 
Karlsson, S., and M. Löthgren (2000). On the Power and Interpretation of Panel Unit Root Tests. Economics Letters, Vol. 66, pp. 249-255.

Kim, H., K-Y. Oh and C-W. Jeong (2005). Panel Cointegration Results on International Capital Mobility in Asian Economies. Journal of International Money and Finance, Vol. 24, pp. 71-82.

Levin, A., C. F. Lin and J. Chu (2002). Unit Root Tests in Panel Data: Asymptotic and Finite Sample Properties. Journal of Econometrics, Vol. 98, pp. 1-24.

Maddala, G. S., and S. Wu (1999). A Comparative Study of Unit Root Tests with Panel Data and a New Simple Test. Oxford Bulletin of Economics and Statistics, Vol. 61, pp. 631-652.

Moon, H. R., and B. Perron (2004). Testing for a Unit Root in Panels with Dynamic Factors. Journal of Econometrics, Vol. 122, pp. 81-126.

Newey, W., and K. West (1994). Autocovariance Lag Selection in Covariance Matrix Estimation. Review of Economic Studies, Vol. 61, pp. 613-653.

Ng, S., and P. Perron (1995). Unit Root Test in ARMA Models with DataDependent Methods for the Selection of the Truncation Lag. Journal of the American Statistical Association, Vol. 90, pp. 268-281.

O'Connell, P. (1998). The Overvaluation of Purchasing Power Parity. Journal of International Economics, Vol. 44, pp. 1-19.

Park, J. Y., and P. B. C. Phillips (1988). Statistical Inference in Regressions with Integrated Processes: Part 1. Econometric Theory, Vol. 4, pp. 468497.

Phillips, P. C. B., and S. Ouliaris (1990). Asymptotic Properties of Residual Based Tests for Cointegration. Econometrica, Vol. 58, pp. 165-193.

Phillips, P. C. B., and P. Perron (1988). Testing for a Unit Root in Time Series Regression. Biometrica, Vol. 75, pp. 335-346

Said, S. E., and D. A. Dickey (1984). Testing for Unit Roots in AutoregressiveMoving Average Processes of Unknown Order. Biometrica, Vol. 71, pp. 599-607. 